\title{
REFERENCE EIGEN-ENVIRONMENT AND SPEAKER WEIGHTING FOR ROBUST SPEECH RECOGNITION
}

\author{
Yuan-Fu Liao, Hung-Hsiang Fang and Chih-Min Yang \\ Department of Electronic Engineering, National Taipei University of Technology, Taipei 106 \\ yfliao@ntut.edu.tw, http://www.ntut.edu.tw/ yfliao
}

\begin{abstract}
In this paper a reference eigen-environment and speaker weighting (RESW) method is proposed for online HMM adaptation. RESW establishes multiple eigen-MLLR subspaces as the set of a priori knowledge according to certain affecting factors, such as noise type, SNR, male and female. It then projects an input test utterance simultaneously into the set of eigen-subspaces and optimally synthesizes out a set of suitable HMMs. The proposed RESW was evaluated on Aurora 2 multicondition training task. Experimental results showed that average word error rate (WER) of $6.11 \%$ was achieved. RESW not only outperformed the multi-condition training baseline (Multi-Con., 13.72\%) but also the blind ETSI advanced DSR front-end (ETSI-Adv., 8.65\%) and the histogram equalization (HEQ, 8.66\%) and the non-blind reference model weighting (RMW, 7.29\%) and Eigen-MLLR (6.14\%) approaches.
\end{abstract}

Index Terms - robust speech recognition. eigen-MLLR, reference model weighting

\section{INTRODUCTION}

Robustness of automatic speech recognition (ASR) to various noisy environments and speakers is the most important issue for mass deployment of ASR-based applications. To this end, online (using only the input test utterance) environment and speaker adaptation methods are often applied to partially eliminate the noise and speaker effect.

Among many possible approaches, a priori knowledgebased methods are often preferred, because they can assist ASR in better dealing with the distortion of a specific noisy environment and speaker. This is especially true for the case of online c since only one utterance from the unknown test noisy environment and speaker is available. These a priori knowledge-based methods usually collect a large set of a priori noisy environment or speaker knowledge and prepare a single group of clusters or a high dimensional eigen-subspace.

For example, piecewise-linear transformation (PLT) [1] and Maximum Likelihood based Stochastic Vector Mappi (ML-SVM) [2] noise compensation methods cluster various types of noisy speech under a variety of SNR conditions and trains one set of HMMs or transformation functions, respectively, for each cluster. Eigen-voice [3] and eigen-MLLR [4] speaker adaptation approaches collect and analyze a set of speaker super-vectors formed by concatenating the set of the mean vectors of the corresponding speaker-dependent HMMs or MLLR transformation matrices, respectively. On the other hand, reference model weighting (RMW) [5], eigen-MLLR [6] and Ensemble speaker and speaking environment modeling (ESSEM) [7] simultaneously consider noise and speaker effect and generate an ensemble speaker and speaking environment super-vectors.

However, it is generally difficult (if not impossible) to prepare a complete set of a priori knowledge, since the number of potential noisy environments and speakers is quite large.

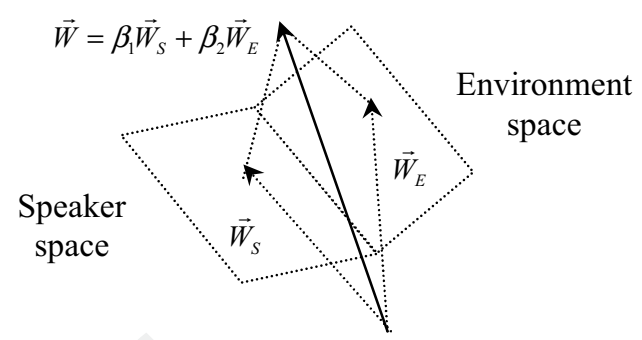

Fig. 1: In RESW, an environment- and speaker-dependent MLLR HMM model transformation super-vector is assumed to be the weighting sum of environment- and speaker-dependent MLLR super-vectors.

To efficiently take the advantage of a priori noisy environment and speaker knowledge, a reference eigenenvironment and speaker weighting (RESW) method is proposed in this paper. The basic idea is shown in Fig.1. RESW assumes that an environment- and speaker-dependent MLLR super-vector could be approximated by the weighting summation of MLLR transformation super-vectors. It therefore decompose the large speaker and environment space to construct multiple smaller eigen-MLLR spaces according to certain affecting factors (maybe not independent), such as noise type, SNR, male and female. RESW then simultaneously projects an unknown test speech to all affecting factordependent eigen-MLLR subspaces and optimally interpolates (in the sense of maximum likelihood criterion) out a set of suitable HMMs.

The benefit by doing this is that (1) a priori knowledge in one set of condition may be reused in others; (2) principle component analysis (PCA) on each condition may reveal specific characteristics and (3) the sparse adaptation data problem may be alleviated. It also is worth noting that RESW method is different with the factor analysis [8] approach which is very successful in speaker verification tasks. The main reason is that RESW considers the distortion of additive noises but factor analysis deal with the convolution channel effects. In other words, in MFCC domain, channel effect could be treated as an additive vector to speaker's one, but noise may also produce some nonlinear distortion.

The paper is organized as follows. Section II describes the proposed RESW method in detail. Section III reports the analysis and experimental results evaluated on the multicondition training task of Aurora 2 corpus [9]. Some conclusions are drawn in the last section.

\section{THE PROPOSED RESW APPRAOCH}

Fig. 2: displays a block diagram of the proposed RESW environment and speaker compensation framework for robust speech recognition. It is operated in two phases: (1) a training phase to analyze and properly organized multiple sets of $a$ priori noisy environment and speaker knowledge to construct a set of eigen-MLLR subspaces and (2) a test phase to project an 
input test utterance into those eigen-spaces and optimally interpolates out a set of test environment and speakercompensated HMMs.

In the training phase, a noisy environment and speakerindependent HMM, $\Lambda_{n i}$, is first trained from the observed speech feature vectors of all seen noisy environments and speakers. Then, all the training utterances are assigned into one of $F$ (maybe overlap) groups according to certain affecting factors. Then the corresponding $F$ sets of noisy environment and speaker-specific MLLR super-matrices $\left\{W_{f, n}, n=1 \sim N_{f}, f=1 \sim F\right\}$ are estimated.

The sets of MLLR super-matrices $\left\{W_{f, n}, n=1 \sim N_{f}, f=1 \sim F\right\}$ are further analyzed by PCA to construct $F$ eigen-MLLR subspaces represented by the sets of MLLR eigen-matrices $\quad\left\{W_{f, m}^{E}, m=0 \sim M, f=1 \sim F\right\} \quad$ with $W_{f, 0}^{E}=1 / N_{f} \sum_{n=1}^{N_{f}} W_{f, n}$ is the mean super-matrix of each groups. The sets of MLLR eigen-matrices $\left\{W_{f, m}^{E}, m=0 \sim M_{f}, f=1 \sim F\right\}$ and the noisy environment and speaker-independent HMM $\Lambda_{n i}$ are then treated as the a priori noisy environment and speaker knowledge.

In the test phase, maximum likelihood criterion and expectation-maximization (EM) algorithm is utilized to optimally find a set of interpolation weights, $\left\{\hat{\alpha}_{f, m}, \hat{\beta}_{f}, m=0 \sim M_{f}, f=1 \sim F\right\}, \quad$ to combine the sets of MLLR eigen-matrices into $\hat{W}$ as follows:

$$
\hat{W}=\sum_{f=1}^{F} \hat{\beta}_{f} \sum_{m=0}^{M_{f}} \hat{\alpha}_{f, m} W_{f, m}^{E}
$$

where $\hat{\alpha}_{0}=1$. Finally $\hat{W}$ is applied to transform $\Lambda_{n i}$ into $\hat{\Lambda}$ for an unknown test environment and speaker,

In the following subsections, we discuss the two main parts of RESW in detail including (1) eigen-MLLR subspace construction and (2) maximum likelihood interpolation.

\subsection{Eigen-MLLR Subspace Construction}

In the RESW framework, we first employ the MLLR method to measure how to adapt $\Lambda_{n i}$ to the observed feature vectors of a specific seen noisy environment and speaker. The relationship between the mean vector $\mu_{s, k}$ of the $k$-th mixture of the $s$-th state of $\Lambda_{n i}$ and its counterpart $\mu_{f, n, s, k}$ in the adapted HMM, $\Lambda_{f, n}$, of the $n$-th noisy environment and speaker in the $f$-th group can be expressed by

$$
\mu_{f, n, s, k}=A_{f, n, s, k} \cdot \mu_{s, k}+b_{f, n, s, k}
$$

where $A_{f, n, s, k}$ and $b_{f, n, s, k}$ are the mean transformation matrix and the bias vector, respectively. It is noted here that only the mean vectors of the mixture components of $\Lambda_{n i}$ are transformed because they are the most important factors for speech recognition.

$A_{f, n, s, k}$ and $b_{f, n, s, k}$ are then concatenated into a MLLR super-matrix, $W_{f, n}$ and all super-matrices of the $f$-th group are collected together, i.e., $\left\{W_{f, n}=\left[\left(A_{f, n, s, k}, b_{f, n, s, k}\right), s=1 \sim S, k=1 \sim K\right], n=1, \cdots, N_{f}\right\}$.

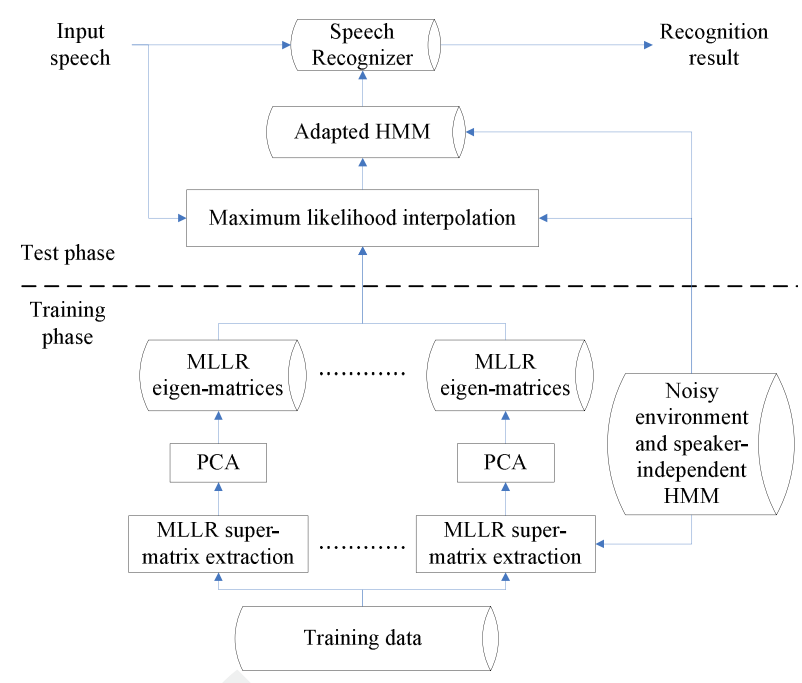

Fig. 2: A block diagram of the proposed RESW fast environment and speaker compensation framework for robust speech recognition.

After that, PCA is applied to analyze and construct an lower dimensional eigen-MLLR subspace, i.e., $\left\{W_{f, m}^{E}=\left[\left(A_{f, m, s, k}^{E}, b_{f, m, s, k}^{E}\right), s=1 \sim S, k=1 \sim K\right], m=0, \cdots, M_{f}\right\}$. And, the entire a priori knowledge of the noisy environment and speaker space is represented by $\Lambda_{n i}$ and the $F$ sets of MLLR eigen-matrices, i.e.,

$$
\left\{\begin{array}{l}
W_{f, m}^{E}=\left[\left(A_{f, m, s, k}^{E}, b_{f, m, s, k}^{E}\right), s=1 \sim S, k=1 \sim K\right], \\
m=0, \cdots, M_{f}, f=1 \sim F
\end{array}\right\}
$$

with $W_{f, 0, s, k}^{E}=1 / N_{f} \sum_{n=1}^{N_{f}} W_{f, n, s, k}$. It is worthy noting that the popular tree structure-based class tying can also be applied to reduce the number of MLLR transformation matrices.

\subsection{Maximum Likelihood Interpolation}

Given the multiple sets of the MLLR eigen-matrices, the noisy environment and speaker-independent HMM $\Lambda_{n i}$, RESW wants to optimally adapted $\Lambda_{n i}$ online to match with the input feature vectors, $O=\{o(1), o(2), \ldots, o(T)\}$, of the unknown test noisy environment and speaker by linearly combining the sets of the MLLR eigen-matrices, i.e.,

$$
\hat{\mu}_{s, k}=\hat{W}_{s, k} \mu_{s, k}=\sum_{f=1}^{F} \hat{\beta}_{f} \sum_{m=0}^{M_{f}} \hat{\alpha}_{f, m} W_{f, m, s, k}^{E} \mu_{s, k}
$$

for $k=1 \sim K$ and $s=1 \sim S$, where $\hat{\mu}_{s, k}, \mu_{s, k}=\left[\begin{array}{ll}\mu_{s, k}^{T} & 1\end{array}\right]^{T}$ and $W_{f, m, s, k}^{E}=\left[\begin{array}{ll}A_{f, m, s, k}^{E} & b_{f, m, s, k}^{E}\end{array}\right]$ are the mismatch-compensated mean vector, the extended mean vector and the augmented mean transformation matrix for the $k$-th mixture component of the $s$-th state of the HMM model, respectively. The input test feature vector sequence, $O=\{o(1), o(2), \ldots, o(T)\}$, can then be recognized using the noisy environment and speaker mismatchcompensated HMM model $\hat{\Lambda}$. 
Let $\Phi=\left[\phi_{1}, \ldots, \phi_{F}, \zeta\right]^{T}$, where $\phi_{f}=\left[\alpha_{f, 0}, \ldots, \alpha_{f, M_{f}}\right]^{T}$, $\alpha_{f, 0}=1$, and $\zeta=\left[\beta_{1}, \ldots, \beta_{F}\right]^{T}$, is the vector of combination weights, the problem can, therefore, be re-stated as: how to optimally estimate the combination weight vector $\Phi$ directly from the observed testing sequence, $O$, in order to adapt the noisy environment/speaker-independent $\mathrm{HMM}, \Lambda_{n i}$, to $\hat{\Lambda}$ for the unknown test noisy environment and speaker. The maximum likelihood (ML) criterion defined below is often adopted for this optimization problem:

$$
\hat{\Phi}=\underset{\Phi}{\arg \max } P(O \mid \hat{\Lambda}(\Phi))
$$

The expectation-maximization (EM) algorithm is applied to solve the optimization problem by taking the state and mixture sequence $\Theta=\{\theta(t)=(s(t), k(t)), t=1, \cdots, T\}$ as the hidden data and add them to form the complete data sequence $\{O, \Theta\}$. An auxiliary function $Q\left(\Phi, \Phi^{\prime}\right)$ is defined as follows:

$$
Q\left(\Phi, \Phi^{\prime}\right)=\sum_{t=1}^{T} \sum_{s=1}^{S} \sum_{k=1}^{K} \gamma_{s, k}(t) \log \left\{c_{s, k} N\left(o(t) ; \hat{\mu}_{s, k}^{\prime}, \Sigma_{s, k}\right)\right\}
$$

where $\Phi$ and $\Phi^{\prime}$ are the old and new MLLR eigen-matrices combination weight vectors, respectively; $\hat{\mu}_{s, k}^{\prime}$ is the mismatch-compensated mean vector of the $k$-th mixture component of the $s$-th state using $\Phi^{\prime}=\left[\phi_{1}^{\prime}, \ldots, \phi_{F}^{\prime}, \zeta_{F}^{\prime}\right]^{T}$; and $\gamma_{s, k}(t)$ is the occupancy probability for $\theta(t)=(s(t), k(t))$ calculated by the forward-backward algorithm using the adapted HMM $\hat{\Lambda}$ derived from $\Lambda_{n i}$ with the old combination weight vector $\Phi$.

By further ignoring some terms not related to $\Phi^{\prime}$, Eq. (6) can be simplified and expressed by

$$
\begin{aligned}
& Q^{\prime}\left(\Phi^{\prime}\right)=-\frac{1}{2} \sum_{t=1}^{T} \sum_{s=1}^{S} \sum_{k=1}^{K} \gamma_{s, k}(t) . \\
& \left\{\left(o(t)-\mathbb{Z}_{s, k} \zeta^{\prime}\right)^{T} \Sigma_{s, k}^{-1}\left(o(t)-\mathbb{Z}_{s, k} \zeta^{\prime}\right)\right\}
\end{aligned}
$$

where

$$
\mathbf{Z}_{f, s, k}=\left\{W_{f, m, s, k}^{E} \mu_{s, k}, m=0 \sim M_{f}\right\}
$$

$\mathbb{Z}_{s, k}=\left\{\mathbf{Z}_{f, s, k} \phi_{f}^{\prime}, f=1 \sim F\right\} \quad$ are the transformed mean matrices.

Then the new weight vector $\Phi^{\prime}$ can be found by solving two linear systems and a close form solution can hence be obtained and expressed by:

$$
\begin{aligned}
\phi_{f}^{\prime}= & \left\{\sum_{t=1}^{T} \sum_{s=1}^{S} \sum_{k=1}^{K} \gamma_{s, k}(t)\left[\beta_{f}^{\prime} \mathbf{Z}_{f, s, k}^{T} \Sigma_{s, k}^{-1} \mathbf{Z}_{f, s, k}\right]\right\}^{-1} \cdot \\
& \sum_{t=1}^{T} \sum_{s=1}^{S} \sum_{k=1}^{K} \gamma_{s, k}(t)\left[\mathbf{Z}_{f, s, k}^{T} \Sigma_{s, k}^{-1}\left(o(t)-\sum_{j \neq f} \beta_{j}^{\prime} \mathbf{Z}_{j, s, k} \phi_{j}^{\prime}\right)\right] \\
\beta_{f}^{\prime}= & \left\{\sum_{t=1}^{T} \sum_{s=1}^{S} \sum_{k=1}^{K} \gamma_{s, k}(t)\left[\mathbb{Z}_{s, k}^{T} \Sigma_{s, k}^{-1} \mathbb{Z}_{s, k}\right]\right\}^{-1} \cdot \\
& \sum_{t=1}^{T} \sum_{s=1}^{S} \sum_{k=1}^{K} \gamma_{s, k}(t)\left[\mathbb{Z}_{s, k}^{T} \Sigma_{s, k}^{-1}\left(o(t)-\sum_{j \neq f} \beta_{j}^{\prime} \mathbf{Z}_{j, s, k} \phi_{j}^{\prime}\right)\right]
\end{aligned}
$$

The expectation and maximization steps of the EM algorithm (i.e., estimate of the occupancy probability, $\gamma_{s, k}(t)$, and calculation of Eq. (8) (9)) are iteratively applied until the likelihood converges.

\section{EXPERIMENTAL RESULTS}

\subsection{Aurora 2 Multi-Condition Training Task}

In the multi-condition training task of Aurora 2 [9] corpus, the eight noises including (1) subway, (2) babble, (3) car, (4) inhibition, (5) restaurant, (6) street, (7) airport and (8) railway, are divided into 4 disjoint sets, i.e., one training and three test sets, sets A, B and C. For the training set, only noise types (1) (4) are available to build the speech recognizer. Test set A also includes noise types (1) (4), but set B has noise types (5) (8). On the other hand, test set $\mathrm{C}$ has the same noise types (1) and (5) but they go through different communication channels compared with the training set. For each type of noise, a set of clean utterances and 5 sets of noisy utterances with SNRs ranging from $0 \sim 20 \mathrm{~dB}$ are used for evaluation.

In all following evaluations, we simply followed the experimental conditions of Aurora 2 protocols.

\subsection{Analysis of Eigen-MLLR Subspaces}

Several distortion affecting factors were considered in RESW including (1) environment (noise type and SNR) and (2) speaker (male and female). All seen noisy environments and speakers were assigned into one of these groups to extract 1 4 sets of a priori noisy environment and speaker-specific MLLR super-matrices (6 MLLR mean transformation matrices, in total 3822 dimensions) and to construct 1 4 affecting factordependent eigen-MLLR subspaces.

Fig. 3(a) and 3(b) shows the projections of the training data on the first two dimensions of the constructed environment and speaker eigen-MLLR subspaces, respectively. From the Fig 3(a), it is interesting to see that the first and second dimension of the first subspace is highly related to the SNR value and noise type, respectively. On the other hand, male and female could be easily separated in the second subspace (Fig. $3(b))$.

\subsection{Performance Evaluation and Discussion}

Several approaches were evaluated including (1) multicondition training baseline, blind (2) ETSI-Adv. [10] and (3) HEQ [11], and non-blind (4) RMW, (5) Eigen-MLLR and (6) the proposed RESW approaches. The difference between the last three methods is that RESW used multiple eigen-subspaces

First of all, Table 1 shows the results of the proposed RESW with different experimental settings. It seems that the speaker factor contributed more than the environmental one. However, it may be different if we remove all the training data with lower SNR $(\leq 0 \mathrm{~dB})$. In other word, dirty training data may pollute the set of a priori knowledge in this case.

Performance comparison and summary are shown in Fig. 4(a) and 4(b). It can be seen from Fig. 4(a) that the blind ETSIAdv. and HEQ methods all performed better than the multicondition training baseline $(13.72 \%)$, with the average WERs of $8.65 \%$ and $8.66 \%$, respectively, were achieved. On the other hand, the non-blind RMW, Eigen-MLLR and RESW further improved the average WERs to $7.29 \%, 6.14 \%$ and $6.12 \%$, respectively.

These results reveal the benefits of utilizing PCAtransformed a priori noisy environment and speaker knowledge Especially, Eigen-MLLR and RESW worked much better than all others on unseen noises and channels, i.e., test set B and C. Moreover, Fig 4(b) also shows that Eigen-MLLR and RESW consistently outperformed others in all different SNR conditions. 
Table 1: Performances of the proposed RESW approach with different experimental settings.

\begin{tabular}{|c|c|c|c|}
\hline \multirow{2}{*}{$\begin{array}{c}\text { \#. of } \\
\text { subspaces }\end{array}$} & \multicolumn{2}{|c|}{ \#.of eigen-vectors } & \multirow{2}{*}{$\begin{array}{c}\text { Error rate } \\
(\%)\end{array}$} \\
\cline { 2 - 3 } & $\begin{array}{c}\text { Environment } \\
\text { (SNR,type) }\end{array}$ & $\begin{array}{c}\text { Speaker } \\
\text { (male,female) }\end{array}$ & 7.04 \\
\hline 1 & 28 & 0 & 6.80 \\
\hline 1 & 24 & 0 & 6.29 \\
\hline 1 & 0 & 110 & 6.18 \\
\hline 1 & 0 & 85 & 6.09 \\
\hline 1 & 0 & 60 & 6.17 \\
\hline 2 & 24 & 60 & 6.12 \\
\hline 3 & 24 & $(55,55)$ & 6.12 \\
\hline 4 & $(6,4)$ & $(55,55)$ & \\
\hline
\end{tabular}

\section{CONCLUSIONS}

In this paper a RESW framework is proposed for fast noisy environment and speaker compensation. Experimental results on Aurora 2 database showed that (1) the distortion characteristics of different kinds of noisy environments and speakers could be separately organized using multiple eigenMLLR subspaces; (2) RESW could efficiently deal with both seen and unseen noisy environments/speakers.

\section{ACKNOWLEDGMENT}

This work was supported by the National Science Council, Taiwan, under the project with contract NSC 96-2221-E-027100-MY2.

\section{REFERENCES}

[1] Zhipeng Zhang, Toshiaki Sugimura and Sadaoki Furui, "A TreeStructured Clustering Method Integrating Noise and SNR for Piecewise Linear-Transformation-Based Noise Adaptation," ICASSP'2004.

[2] D. Zhu and Q. Huo, "A Maximum Likelihood Approach to Unsupervised Online Adaptation of Stochastic Vector Mapping Function for Robust Speech Recognition," in Proc. ICASSP 2007

[3] R. Kuhn, et. Al., "Eigenvoices for Speaker Adaptation," Proc. ICSLP'98, pp.1771-1774, 1998

[4] Kuan-ting Chen, Wen-wei Liau, Hsin-min Wang and Lin-shan Lee, "Fast Speaker Adaptation using Eigenspace-Based Maximum Likelihood Linear Regression," Proc. ICSLP 2000, pp. 742-745, Bejing, China, October 2000.

[5] Yuan-Fu Liao, Jyh-Her Yang, Chi-Hui Hsu, Cheng-Chang Lee and Jing-Teng Zeng, "A Reference Model Weighting-based Method for Robust Speech Recognition”, InterSpeech'2007

[6] Yuan-Fu Liao, Hung-Hsiang Fang and Chi-Hui Hsu, "EigenMLLR Environment/Speaker Compensation for Robust Speech Recognition", InterSpeech'2008

[7] Yu Tsao and Chin-Hui Lee, "An Ensemble Modeling Approach to Joint Characterization of Speaker and Speaking Environments", InterSpeech'2007

[8] P. Kenny, G. Boulianne, P. Ouellet, and P. Dumouchel, "Joint factor analysis versus eigenchannels in speaker recognition," IEEE Transactions on Audio, Speech and Language Processing, vol. 15, no. 4, pp.1435-1447, 2007

[9] Aurora project database 2- evolution package, http://www.elda.org/

[10] ETSI ES 202212 v1.1.1 2003, "Speech Processing, Transmission and Quality Aspects(STQ); Distributed speech recognition; Extended advanced front-end feature extraction algorithm; Compression algorithm; Back-end speech reconstruction algorithm"

[11] Á. Torre, A. Peinado, J. Segura, J. Pérez-Córdoba, Ma Carmen Benítez and A. Rubio, "Histogram Equalization of Speech Representation for Robust Speech Recognition," IEEE Transactions on Speech And Audio Proc., Vol. 13, No. 3, May 2005.

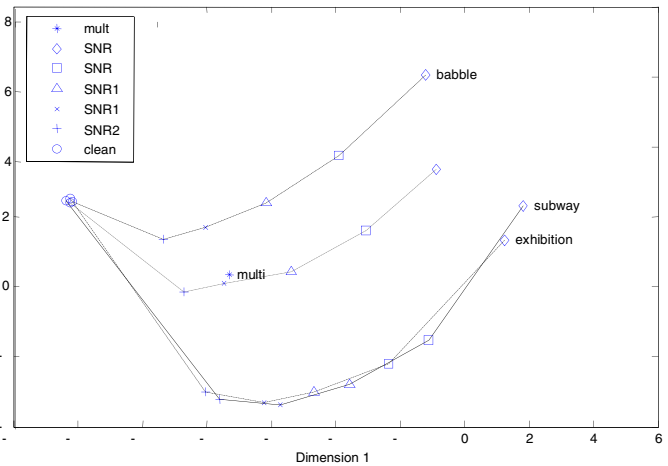

(a)

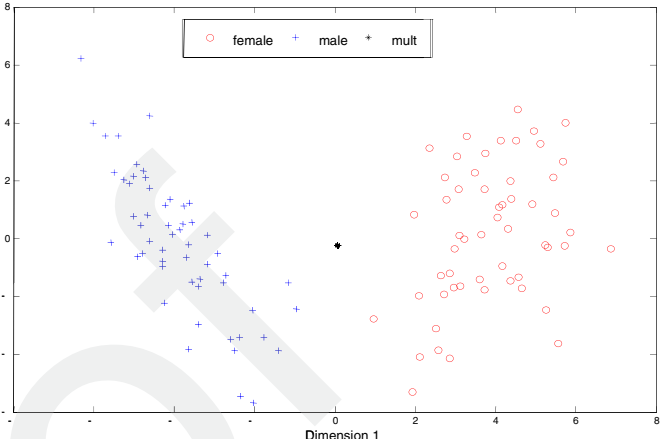

(b)

Fig. 3: The constructed environment and speaker eigen-MLLR subspaces and the projections of super-matrices of various noisy environments and speakers in Aurora 2 multi-condition training set: (a) four types of noises and (b) female (circle) and male (plus) in the first two dimension of the environment and speaker eigen-MLLR subspace, respectively.

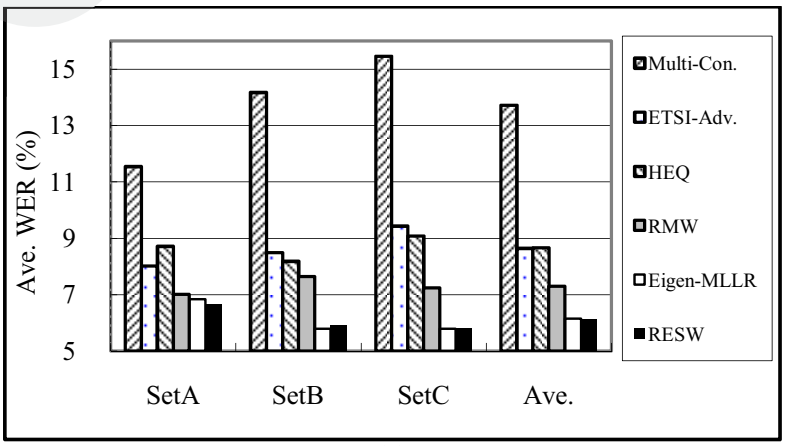

\begin{tabular}{|c|c|c|c|c|c|c|}
\hline \multicolumn{7}{|c|}{ (a) } \\
\hline $\begin{array}{l}\text { SNR } \\
(\mathrm{dB})\end{array}$ & $\begin{array}{l}\text { Multi- } \\
\text { Con. }\end{array}$ & $\begin{array}{c}\text { ETSI- } \\
\text { Adv. }\end{array}$ & HEQ & RMW & $\begin{array}{l}\text { Eigen- } \\
\text { MLLR }\end{array}$ & RESW \\
\hline clean & 1.35 & 0.98 & 1.21 & 1.20 & 1.06 & 1.03 \\
\hline 20 & 2.47 & 1.55 & 1.42 & 1.25 & 0.97 & 0.98 \\
\hline 15 & 4.05 & 2.25 & 2.15 & 1.83 & 1.41 & 1.32 \\
\hline 10 & 6.38 & 4.17 & 3.76 & 3.21 & 2.37 & 2.41 \\
\hline 5 & 14.53 & 9.44 & 9.00 & 7.76 & 6.13 & 6.07 \\
\hline 0 & 41.73 & 25.82 & 26.95 & 22.43 & 19.82 & 19.80 \\
\hline
\end{tabular}

(b)

Fig. 4: Performance comparison of various approaches on Aurora 2 multi-condition task including (1) multi-condition baseline, (2) ETST advanced front-end (ETSI-Adv.), (3) histogram equalization (HEQ), (4) reference eigen-environment and speaker weighting (RESW): (a) average performance over $0 \sim 20 \mathrm{~dB}$ SNRs on different test sets and (b) average performance over test set $\mathrm{A} \sim \mathrm{C}$ on different SNRs. 